\title{
Hipertensión arterial en una unidad de cuidados intensivos (UCI) neonatal
}

\author{
Dra, Colomba Norero V. ${ }^{1}$ : Dra. Amalia Concha N. ${ }^{2}$; Dra. IJizabeth Hubach K. ${ }^{3}$; \\ Sr. Jorge Galdámez V.
}

Arterial hypertension among admissions to intensive neonatal care

An experience on arterial hypertension in neonates is presented. This clinical entity was found in $2.5 \%(6 / 234)$ of the admissions to an intensive care neosatal unit algong a six months period. In two patients arterial hypertension was attributed to acute renal fuilure due to renal vascular thrombosis originated in multiple concomitant conditions and umbilical artery catheterization. Other two cases caried complex congenital malformations of the urinary tract associated to renal dysplastic elements. One patient had aortic coaretation of difficult medical management and he was subinitted to surgical repair with a smooth outcome. Finally in the last case a subcndocardic fibroelastoxis without associated coarctation was described at necropsy. The case fatality ratio was $50 \%(3 / 6)$.

(Key words: Arterial hypertension, neonates, newborn).

La detección de hipertensión arterial (HA) en el Recién Nacido (RN) ha ido en aumento en estos últimos años por la incorporación rutinaria de la medición de la presión arterial (PA) en los niños de al to riesgo.

1. Profesor Titular de Pediatria. Servicio y Depto. de Pediatría. División Ciencias Médicas Occidente. Facultad de Medicina, Univer sidud de Chile.

2. Unidad de Neonatología, Servicio de Pediatría, Hospital San Juan de Dios.

3. Becario Departamento de Pediatría, División Ciencias Médicas Occidente, Facultad de Medicina, Unjversidad de Chile.

4. Interno Departamento de Pediatría, División Ciencias Médicas Occidente, Facultad de Medicina, Univer sidad de Chile.
El aumento de la sobrevida de este grupo de $\mathrm{RN}$, con los procedimientos invasivos que implica su monitoria; las frecuentes complicaciones de. rivadas de la inestabilidad cardiova scular, la facilidad de adquirir infecciones graves, $y$ el uso de múltiples tratamientos farmacológicos son algunas de las causas que aumentan la incidencia de HA.

La ausencia de publicaciones nacionales en relación a este tema nos ha motivado a presentar nuestra experiencia en una Unidad de Cuidados Intensivos (UCI) de Neonatología que recibe un gran número de ingresos de alto riesgo.

\section{MATERIAL Y METODO}

Se presenta el estudio retrospectivo de 234 RN 
hospitalizados en UCl de Neonatologia de nuestro Hospital. Seis de elks presentaron hipertensión arterial (IAA) (2.5\% de los ingresos de LCI neothata).

Sc considetó que los pacientes ten ian HA cuando li presión arterial sitólica (PAS) medida con Doppler4,5,6 fue superior a 70 nाmHg en recićn nacidos pre-término (RNPT) ${ }^{1,2}$ o mayor a $90 \mathrm{~mm} / \mathrm{Hg}$ en recién nacidos de tértnino (RNT) ${ }^{3}$ repetida en varias ocasiones.

\section{RESULTADOS}

Las caracter ísticas clínicas y la edad de aparición de HA se presentan en la Tabla 1. El diagnóstico y la evolución se aprecian en la Tabla 2.

Descripción de los pacientes:

Caso 1. RNPT AEG PN: $1.480 \mathrm{gr}$. Sufrió sindrome de dificultad respiratoria idiopática (SDRI) y Bronconeumonia (Br.V). Fue conectado a respirador en las primeras horas de vida, cateterizándose arteria umbilical. Al quinto dia por signos de ductus permeable con insuficiencia cardíaca recibió digitálicos y 3 dosis de Indome. tacina en coincidencia con to cual se observó aumento de la creatininemia y oliguria transito- ria. A los 10 días se detectó aumento unilateral de tamaño renal, mayor elevación de la creatininemia $y$ aumento progresivo de la PA. Desde e] día siguiente al retio del catéter de arteria umbilical (dia 12) la PA y la creatinina siguieron elevándose hasta llegar a $120 \mathrm{mmHg}$ y $2,2 \mathrm{mg} \%$ respectivamente a los 18 días, debiendo usarse Diazoxide durante ese periodo. $A$ los 20 dias se comprobó sepsis por Candida albicans. Falleció a los 35 dias con signos de insuficiencia circulatoria periférica, y persistencia de cifras moderadamente elevadas de PA. La necropsia demostró comunicación interauricular, ate]ectasia masiva y signos de shock.

Caso 2. RNPT AEG PN: 2.560 gr. Cesárea. Se instala ventilación mecánica y catéter umbilical por SDRI. $\Lambda 190$ día, en coincidencia con deterioro clínico detectó hemorragia puImonar, aumento de támaño del rin̄ón izquierdo, orina rosada y moderado aumento de PA ( $80 \mathrm{~mm} \mathrm{Hg}$ ). Los cultivos fueron positivos, con Estafilocoes aureus multirresistente, en secreción traqueal y catéter umbilical. En los urocultivos se aisló Klebsiella. Recibió varios antibióticos. A los 17

Tabla 1. Hipertensión arterial en recién nacidos: caracterist jos clinicas.

\begin{tabular}{|c|c|c|c|c|}
\hline Pacionte & Sexo & Peno Nate & $\begin{array}{l}\text { Cifias temsiouales } \\
\text { máx imas (Doppler) }\end{array}$ & $\begin{array}{l}\text { Aparición de } \mathrm{HA} \\
\text { en días de vida }\end{array}$ \\
\hline 1 & $\mathbf{M}$ & 1480 RNPT & 120 & 11 \\
\hline 2 & $\mathbf{M}$ & 2560 RNPT & 140 & 9 \\
\hline 3 & $M$ & 2950 RNT & 120 & 5 \\
\hline 4 & $\mathrm{M}$ & $2750 \mathrm{RNT}$ & 130 & 8 \\
\hline$\xi$ & $\mathbf{M}$ & $3500 \mathrm{RXT}$ & 180 & 28 \\
\hline 6 & $\mathrm{M}$ & $3310 \mathrm{R} \times{ }^{*} \mathrm{~T}$ & 220 & 15 \\
\hline
\end{tabular}

Tabla 2. Evolución de 6 tecién nacidos con hipertensión ar terial.

\begin{tabular}{|c|c|c|c|}
\hline Paciente & Diagnóstico & $\begin{array}{l}\text { PA máx. } \\
\text { Doppler }\end{array}$ & Evolución \\
\hline 1 & $\begin{array}{l}\text { Insuf. renal } \\
\text { Trombosis vascular } \\
\text { SDR, BrN, Ductus Sepsis }\end{array}$ & 120 & $\begin{array}{l}\text { Fallece } 35 \text { dias de vida } \\
\text { Necrop.: CIA, Atelectasia } \\
\text { masiva, Signos de shock }\end{array}$ \\
\hline 2 & $\begin{array}{l}\text { Insuf. rena! } \\
\text { Trombosis vascular } \\
\text { SDR. BrN, Sepsis }\end{array}$ & 140 & $\begin{array}{l}\text { Controi a los } 3 \text { meses } \\
\text { Cilíndros hialinos } \\
\text { PA norma! }\end{array}$ \\
\hline 3 & $\begin{array}{l}\text { Displasia tenal quística. } \\
\text { Malf. uretral }\end{array}$ & 120 & $\begin{array}{l}\text { Fallece } 21 / 2 \text { meses de vida } \\
\text { Insuf. renal crónica }\end{array}$ \\
\hline 4 & $\begin{array}{l}\text { Agenesia tenal der. } \\
\text { Hidronefrosis izda. } \\
\text { Valvas uretra }\end{array}$ & 130 & $\begin{array}{l}\text { Op. Vesicotomía } \\
\text { Control UTI } \\
\text { PA normal }\end{array}$ \\
\hline 5 & Coar tación aórtica & 380 & Op. En control. PA normal \\
\hline 6 & $\begin{array}{l}\text { Fibroetastosis sub- } \\
\text { endocárdica } \\
\text { ¿Coartación aórtica? }\end{array}$ & 220 & $\begin{array}{l}\text { Fallece a los } 3 \text { meses } \\
\text { BrN, Insuf. cardiaca } \\
\text { Necrop.: Fibroelastosis } \\
\text { subendocárdica. No se } \\
\text { describe coartación aórtica. }\end{array}$ \\
\hline
\end{tabular}


días de vida se detectó creatininemia de $1,76 \mathrm{mg} \%$ y PAS de $140 \mathrm{~mm} H \mathrm{H}$. Continuó con PA eleqada durante I mes. Se efectuó ecografía y pielografía a los 20 dias que fueron normales. $A$ los 50 días fue dada de alta siendo controlado hasta los 5,5 meses con nueva pielografía normal, $P A$ normal y vajias infecciones urinarias.

Caso 3. RNT AEG PN: 2,950 gr. Cesárea a las 38 semanas de gestación por cesarea anterior; oligoamnios severo y presentación podálica. Tenia pie bot, pene largo con bolsa escrotal comunicada a la uretra $y$ se palpaban 2 masas abdominales, en el lado jzquierdo. que se interpretaron como riñón y uréter. Creatininemis al 20 día de $8 \mathrm{mg} \%$. Su PAS era de $120 \mathrm{mmHg}$ a $l o s$ 6 dias y se redujo a $85 \mathrm{~mm}$ después de recibir metildopa. Signos de reacción ventricular izquierda al electrocardiograma. La ecografía abdominal demostró gran hidronefrosis y un uréter flexuoso a jzquierda, sın visualizarse riñón derecho tij vejiga. No se logró identificar riñones en la pielografía a las 6 horas de inyección de medio de contraste y la uretrocistografía no mostró reflujo vesicoureteral. Cuando tenía l mes de edad se realizó una ureterostomia culánea izquierda para descomprinir la via urinaria y una biopsia renal que demostró displasia renal microquistica. Falleció a los 2,5 meses en insuficiencia renal crónica.

Caso 4. RNT AEG PN: 2.750 gr. Fórceps a las 38 sema nas de edad gestacional por sufrimien. to fetal. Al 3er. día de vida presentaba hiperbilirrubinemia y se palpó una masa abdominal izquierda. El estudio basado en ecografia abdominal, cintigrafia renal y pielografía de eliminación $y$ ureterocistografía concluyó en hidrone. frosis izquierda, agenesia renal derecha y valvas uretrales. Al $8^{\circ}$ dia de vida su PAS era de $130 \mathrm{mmHg}$; presentaba hiperkalemia y creatininemia de $1.13 \mathrm{mg} \%$; por lo que a los 13 dias de vida se decidió descomprimir la vía urinaria mediante vesicostomía. Evolucionó con infecciones urinarias at repetición y $\mathrm{BrN}$, por lo que recibe diferentes antibióticos. La creatininemia y PA se. normalizaron $y$ fue dado de alta a los 45 días de edad. Al cumplir 1 año se efectuó cierte de la vesicostomía y resección de las valvas uretrales. En tjempo posterior se hizo pielostomía cutănea con el propósito de reparar el resto de la vía urinaria. Ha cursado sin hipertensión ni retención nitrogenada.

Caso 5. RNT AEG PN: 3.500 gr. Cesárea por malas condiciones maternas. Periodo de RN normal. Ingresó a la edad de 28 días con insuficiencia cardíaca, bronconeunonia, atelectasia lóbulo superior izquierdo e hiperfensión arterial (180/80 - $0 \mathrm{~mm} \mathbf{H g})$ en las extremidades superiores y PAS de $50 \mathrm{mmHg}$ en las inferiores.
Habiéndose demostrado una coartación aórtica fue tratado con pobres resultados con Furosemida, Captopril y Metildopa por lo que debió ser operado a los 2 meses de edad. La evolución posterior fue satisfactoria con PA normal.

Caso 6. RNT AEG PN: 3.310 gr. Cesáréa por distocia de posición. Periodo neonatal normal. A la edad de 15 días ingresó en insuficiencia cardiaca con PAS de $105 \mathrm{~mm} H \mathrm{Hg}$. Pulsos periféricos palpables en las 4 extremidades. La ecografía abdominal, la pielografía de eliminación y la creatininemia eran normales, pero habia cardiomegalia por crecimiento de ambas cavidades izquierdas en la radiografia de tórax, evidencia ecográfica de hipertrofia concéntrica del ventrículo jzquierdo $y$, en el electrocardiograma, hipertrofia ventricular izquierda $y$ bloqueo atrioventricular. A los 30 dias su PA era de $110 / 70 \mathrm{mmHg}$ en extremidades superiores $y$ $75 / 40 \mathrm{mmHg}$ en las inferiores. abandonó el control reingresado a los 3 meses de edad en jusu. ficiencia cardjaca con PAS de 220 mmila en las extremidades superiores. Falleció a pesar del tratamiento con diazoxide, metildopa y diuré. ticos. En la attopsia solo se describió fibroelastosis subendocárdica.

\section{COMENTARIO}

La frecuencia de $2,5 \%$ de HA en los ingresos de la UCI de Neonatología del Hospital San Juan de Dios en el I Semestre de 1985 es semejante a la encontrada en varios trabajos de la literatu$\mathrm{rat}^{7,8,9}$.

Del total de nuestros 6 pacienres que presentaron $\mathrm{HA}$ en el período de $\mathrm{RN}, 2$ correspondieron a malformación del parénquima renal asociada a uropatía obstructiva y 4 a patología vascular renal ( 2 pac.) y extrartenal (2 pac.).

La trombosis de la arteria renal por la cateterización de la arteria umbilical se describe como la causa más frecuente de HA en esta etapa de la vida. Su comprobación defínitiva sólo puede hacerse por medio de aortografia, estimándose que su frecuencia real es mucho más aita que la que se expresa clínicamente24. Desde el punto de vista clínico se estima que alrededor de $3 \%$ de las cateterizaciones de la arteria umbilicat desarrollarán $\mathrm{HA}$ por trombosis de la arteria renal. Esta observación se ha avalado en nuestro país por la experiencia de Tapia y colaboradores $^{13}$ que encuentran HA transitoria en $2,54 \%$ de los RN con cateterización de la arteria umbilical. Según estos autores la presencia de complicaciones tromboembólicas no se correlacionó con la calocación alta o baja del catéter, cosa que no 
concuerda con la literatura sobre el tema ${ }^{11}$; asimismo relacionan la $\mathrm{HA}$ con la duración del cateterismo lo que tampoco se describe en la experiencia de otros autores.

En la UCl de Neonatología del Hospital San Juan de Dios se efectúan como promedio 40 cateterizacjones umbilicales anuales, debiendo encontrarse según los datos analizados, alrededor de 2 casos de $\mathrm{H}$ A secundaria a trombosis de arteria renal por año.

Los pacientes 1 y 2 en que puede plantearse clínicamente dicho diagnóstico comparten características comunes: RNPT con sindrome de distress respiratorio grave que requirieron ventilación mecánica, cateterización de arteria umbilical y que posteriormente presentaron sepsis a Gram(...); debiendo utilizarse aminoglicósidos. Además uno de ellos presentó reapertura del ductus que requirió 3 dosis de Indometacina. Todos estos factores, per se, explican la presencia de $\mathrm{FRA}^{15}$. La asociación con HA plantéa la posibilidad de fenómenos tromboembólicos, probablemente arteriales, dada su mayor frecuencia en los casos de catcterización de arteria umbilical.

Mientras el Caso 1 parece corresponder a dicha patología, el caso 2 nos merece algunas dudas, puesto que la $\mathrm{HA}$ se desarrolla a los 17 días de vida (ya retirado el catéter umbilical) concomitantemente con aumento brusco de tamaño renal, hematuria y alza de creatininemia que se presenta en pleno períado séptico. Esto nos hace plantear más bien una trombosis venosa. La pielografía normal a los 19 dias no descarta dicho diagnóstico ${ }^{17}$. Si bien la presencia de $\mathrm{HA}$ por trombosis venosa es francamente menos frecuente ${ }^{4}$ dicho diagnóstico debe ser considerado en un $\mathrm{RN}$ con las características del Caso 2. Podría aceptarse la posibilidad de una trombosis de arteria renal en los primeros días de evolución.

$\mathrm{La}$ imposibilidad en nuestro medio de efectuar aortografía impidió una confirmación diagnóstica. Se ha descrito que el renograma radioisotópico puede contribujr al diagnóstico de trombosis de arteria renal ${ }^{24}$.

Los pacientes 3 y 4 correspondieron a malformaciones complejas de la via urinaria. En la literatura ${ }^{18,19}$ se describe asociacion de elementos displásicos del parénquima renal en las uropatías obstructivas tal como lo comprobamos en el RN 3, y es altamente probable en el paciente 4 (portador de valvas uretrales y agenesia renal unilateral). En ambos RN los procedimientos quirúrgicos descompresivos de la vía urinaria llevaron a una normalización de la PA

Dos de nuestros pacientes correspondieron a patología cardiovascular. La coartación aórtica es la causa más frecuente de $\mathrm{HA}$ a cualquier edad pediätrica $20,21,22$, siendo bastante excepcional que se manifieste clínicamente durante el periodo neonatal inmediato. Nuestro Caso 5 se presentó como una Insuficiencia Cardíaca a los 28 dias de vida. Es probable que una determinación de la PA en los dias previos hubiese detectado cifras tensionales elcvadas. La refractariedad al tratamiento farmacológico en este enfermo obligó a la corrección quirúrgica a los 2 meses de vidi.

Nuestro 60 paciente ingresó a los 15 días de vida en Insuficiencia Cardiaca, comprobándose cifras moderadamente elevadas de PA con marcada diferencia tensional entre extremidades superiores e inferiores. E1 estudio ecocardiográfico no fue concluyente y se dio de alta sin diagnóstico etiológico. No se controló y reingresó a los 3 meses con cifras muy elevadas de PA que no respondieron al tratamiento. En la necropsia se comprobó una fibroeleastosis subendocárdica (Caso 6). Es frecuente en estos casos, la asocid. ción con coartación aórtica ${ }^{22}$ lo que contribuye a explicar la HA. En nuestro pacjente no se relató dicho hallazgo en la Anatomía Patológica, aunque la clínica del paciente es sugerente de dicha patología.

Det total de nuestros pacientes fallecieron 3 $(50 \%)$, I a los 3 meses de vida (Caso 6) sin recibir tratamiento ni controles intermedios, pero debido a su patología hipertensiva; un segundo paciente falleció en insuficiencia renal crónica a los 2.]/2 meses (Caso 3 ) y por último el Caso I falleció a los 35 días de vida por compromiso pulmonar y shock.

En relación a pronósticos, la $\mathbf{H A}$ por trombosis de arteria renal se describe como transitoria en la mayor parte de los pacientes pero faltan estudios prospectivos para analizar el estado funcional renal residual y la probable reaparición de HA en edades posteriores en dichos pacientes.

En los RN con malformaciones renales com. plejas y uropatía obstructiva los procedimientos quirúrgicos descompresivos pueden contribuir a mejorar las cifras tensionales. Sin embargo las alteraciones de desarrollo parenquimatoso renal, especialmente el riñón poliquistico de tipo infantil se acompañan de $\mathrm{HA}$ desde el periodo de RN extraordinariamente severas y refractarjas al tratamiento, como es nuestira experiencia en el tema ${ }^{23}$. En el periodo de observación analizado no tuvimos ningún paciente portador de dicha patologia.

Podemos conclujr que la presencia de $\mathrm{HA}$ en el RN nos está indicando, en un alto porcentaje de casos, graves patologías dè base que están condicionando la presencia de cifras tensionales elevadas como un componente más del cuadro clínico del RN. 


\section{RESUMEN}

Se presenta la experiencia de HA en utta Unidad de Cuidados Intensivos Neonatológica. correspondienda a] 2,5\% de los ingresos en un período de observación de 6 meses $(6 / 234$ hospitalizados). Se comprueba en 2 pacientes insuficiencia renal aguda, probablemente por trombosis vascular renal desencadenada por múltiples factores y asociada a cateterización de arteria umbilical. Otros 2 RN presentaron malformaciones complejas de la via urinaria, asociada a elementos displísticos del parénquima renal. Un paciente presentó coartación aórtica de dificil tratamiento médico y el último una fibroeleastosis subendocárdica sin coartación asociada comprobada en necropsia. Fallecen 3/6 pacien. tes.

\section{REFERENCIAS}

1. De Siet, M., Fayers, P., Shinebourne, E. Systolic Blond Pressure in a Population of Intants in the tirst year of life: The Brompton Sudy. Pediatrics. 65: $1028,1980$.

2. Versmold, H., Kitternan, J, Phibbs, R. er al: Aort.jc blood pressure during the first 12 hours of life in infants with birth weight 610 to 4220 gr. Pediatrics. 67: 60 ? 1981 .

3. Lagler, U., Duc, G.: Systolic blood pressure in normal newborn infants during the first 6 hours of life. Transcutaneous Doppler Ultrasonic Teechnique. Biol. Neonate. 37: 243, 1980

4. Norero, $C_{\text {. }}$ Hiperternsión Veonatal. Actualización. Rev. Chil. Pediatr. 57:465, 1986.

5. Lum, I.. Jones, $D .:$ The effect of cuif width on systolic blood pressure measurements in neonates. J. Podiatr. 91: 963, 1977.

6. Earley, $A$., Favers, P., Ng. $S$, ef al: Blood pressurs in the first 6 weeks of life. Arch. Dis. Child. 55: 75, 1980.

7. Adehnen, r.: Nconatal Hypertension. Pediatr. Clin. North, Am. 25:99, 1978.

8. Fanaroff, A., Stork, E., Carlo, W.: Neonatal Iupertensión. Fn: Children's Blood Pressuras. Report of 88th Ross Conference on Pediatric Research. Columbus, Ohio, 1985, pp. 85-91.
9. Leder, M. Anmable, W., Kliegman, R.: Hypertensive retinopathy in the newborm infant. J. Pediatr. 103 $781,1983$.

10. Ademan, $R$ : Letter to the editor. Acta Paediatr. Scand, 70: 413, 1981.

11. Bauer, S., Feldman, S., Gells, S. et al- Nconatal Hypertension. A complication of unbilical artery rathelerization. N. bingl. J. Med. 293: 1032, 1975.

12. Baldwin, Ch, Ifolder, T., A shcraft, $K$. et al: Neondtal Renovascujar Hypertensión. A complication of aortic monitoring catheters. J. Pediatr. Surg. 16: 820,1981 .

13. Tapia. J., Venturo Janca, $P$., Gonzáte2, $H$. et al: Cateterisno umbilical arterial. Scis años de experiencia. Rev. Chil. Pediat T. 57: 133, 1986.

14. Arand, S.: Acute renal failure in rewbors. Pediatr Clin. North. Am. 29: 791, 1982

15. Obevesekere, H. Pankhurst, S., Yu, V.: Pliarmacologrial closure of ductus arteriosus in preterm infants using intometacin. Arch. Dis. Child. 55: 271,1980

16. Rasoulpour, M., Mc Lean, R.: Renal venous thr ombosis in nconates. Am. J. Dis. Clild. 134 276,1980

17. Arneil, $G$. .: Renal venous thrombosis. Contrib Nephrol. 15: 21, 1979.

18. Mathicu, $H$. A tteinte rénale an cours des utopathies malforma tives. Royer, Habjb, Mathieu, Broyer. Ed. Tlammarion 3 a édition. París 1983, pp. 162 J63.

19. Gibou, N, Bartoletti. A., Urizar, R.: Severe hypertension in a newborn associated with increased resin production by a hypoplastic kidney. J. [Jrol. I28: $570,1982$.

20. Baghy, $S$ : Dissection of pathogenetic factors in coarclation hypertension. I:n: NHLBI Workshop on Juvenile Hypertension, Bethesda, Maryland, USA, 1984, pp. 253-266.

21. Mace, S., Hirschfell, S.: Hypertensive enceplatopathy. A cause of neonatal scizunes. Am. J. Dis. Child. 137: 32,1983.

22. Blackburn, Wt: Vascular Pathology in Hypertensive Childen. En NIILBJ Workslop on Juvenile Hypertension, Bcthesda, Maryland, USA, 1984. pp. $335-360$

23. Barrera, M. Quezada, L.: Riñón poliquístico infántil. Rev. Chil. Pediatr, 57: 35, 1986.

24. Adelman, R.: Neonatal hypertension. In NHI.BI Workshop on Jusenile Hypertension, Bethesda, Maryland, May 26 and 27, 1983, Biomedical Information Corporation, NY. 1984. 